\title{
A COMPARISON OF TWO MODAL ANALYSIS METHODS: AN IMPACT HAMMER VERSUS LASER VIBROMETRY
}

\author{
JaKub KRejČÍ* Petr Beneš
}

\begin{abstract}
Brno University of Technology, Faculty of Electrical Engineering and Communication, Department of Control and Instrumentation, Technická 3082/12, Královo Pole, 61600 Brno, Czech Republic

* corresponding author: xkrejc44@stud.feec.vutbr.cz
\end{abstract}

ABSTRACT. This paper presents a comparative experimental-analytical study on the performance of Multi-Input Single-Output (MISO) and Single-Input Multi-Output (SIMO) techniques to identify the modal properties of a cantilever beam. A 2-D laser interferometry was employed to perform the SIMO modal test, while for the MISO test configuration, a conventional accelerometer was used to measure the response of the structure. Comparing the experimentally-measured natural frequencies with those calculated by the FEM model, a maximum difference of $4 \%$ between natural frequencies was observed. The repeatability of both techniques is also investigated in this paper and it is shown that the difference between modal properties identified under different operators is less than $0.5 \%$ for both the MISO and SIMO techniques.

KeYwords: Modal analysis, free beam, 2D interferometer, COMSOL, ModalVIEW.

\section{INTRODUCTION}

Modal analysis is a commonly used procedure to determine mechanical dynamic properties and many modal parameter estimations can be applied [1, 2]. It is feasible to develop analytical models to investigate the dynamic behaviour of simple structures, however, for complex structures, either Finite Element methods (FEM) or experimental modal analysis can be used to estimate/measure the dynamic properties of the structure [3].

Firstly, narrow band analogue filters were applied for the evaluation of modal analysis, but then, FFT analysers became more used owing to the evolution of measurement systems. Nowadays, computers have a high computing performance and a model including modal analysis or other parameters can be performed in a short time and it can be compared with a real measurement [4].

Well known examples of modal analysis can be found in car industry, aero industry or civil engineering. Generally, components are firstly modelled, optimized and then prototypes are tested. The aim is to find a dominant resonance, which can cause bothersome noises or can be crucial for the material endurance. Design improvements or different materials affect the frequency response and key resonances can be damped or shifted to noncritical values [4].

Generally, accelerometers are attached to tested objects to measure their vibrations. In some cases, objects for modal analysis are not large enough and the response has to be measured by other means than by accelerometers, which can significantly influence the frequency response [3. In these cases, an interferometer can be used for non-contact sensing and movement trajectory can be determined in few moments, especially when a 2D scanning option is arranged.
During last years, many publications focused on modal analysis were published to demonstrate its utilization in different areas. For example, one deals with a modal analysis of a robot arm [5], mostly performed by a simulation, a comparison with an experiment is only mentioned, but not provided. Other publication solves similar problematics - modal analysis of cantilever beam [6]. The main comparison is performed between an analytic solution and a model without any measurement. However, a similarity of the results is mentioned, therefore, the simulation is an appropriate method, when an experiment cannot be performed. Other remarkable publications deal with an analysis of a piston [7] or supported beam bridge [8]. This paper will present a complex comparison of an analytic solution, simulation and measurement at a simple object, which results are achievable by all methods.

A more complex article is focused on an analysis on a laboratory test plate [9] and provides a comparison between experimental and operational modal analysis, which showed some small differences. Last example deals with a modal analysis of a beam tendon [10, which is performed both theoretically and experimentally. The results of both methods were comparable. These articles show a continuous activity in the field of modal analysis, but many articles are focused on a comparison of an analytic solution and experiment (but simulation is not performed), or simulation and experiment (without analytic solution, which may be impossible due to the complexity of the object). This study will present two methods of modal analysis (one using an impact hammer and the other using laser vibrometry) of a chosen structure, which will be compared from multiple point of views, such as quality of results, repeatability, price or preparation time. The results will be compared with an analytic solution and 


\begin{tabular}{lcccccc}
\hline $\mathrm{n}$ & 0 & 1 & 2 & 3 & 4 & 5 \\
\hline$\delta_{n} L$ & 0 & 4.7300 & 7.8532 & 10.996 & 14.137 & 17.279 \\
\hline
\end{tabular}

TABLE 1. Solutions of non-trivial equation for free-free beam.

FE results. Then, a laboratory set for students will be prepared to demonstrate this topic.

\section{EQUATION OF MOTION}

Let us consider a regular beam of a length $L$, density $\rho$ and Young's modulus $E$. The basic equation of motion of a beam is [11 13:

$$
E I \frac{\partial^{4} w}{\partial x^{4}}+\rho A \frac{\partial^{2} w}{\partial t^{2}}=0
$$

where $I$ is area moment of the beam cross section, $w$ transverse displacement, $A$ area of cross section and $t$ is time. Parameters $I$ and $A$ are obtained from dimensions:

$$
\begin{aligned}
I & =\frac{b h^{2}}{12} \\
A & =b h
\end{aligned}
$$

where $b$ is the width and $h$ the height of the beam. A solution for a free-free beam is formulated by nontrivial equation [11]:

$$
\cos \delta_{n} \cosh \delta_{n} L=1
$$

where $\delta_{n}$ is n-th root of the equation.

First five solutions of the equation are shown in Table 1

With this partial results, the natural frequencies of a beam can be determined by equation:

$$
\begin{gathered}
\omega_{n}=\delta^{2} \sqrt{\frac{E I}{\rho A}} \\
f=\frac{\omega}{2 \pi}
\end{gathered}
$$

If it is necessary to compute frequencies in orthogonal direction, beam length $L$ is replaced with the beam width. Other non-trivial modes cannot be determined by this equation, for example, the beam can move in a diagonal direction. It is impossible to specify complex nature frequencies with presented equations, the boundary conditions for the whole equation (4) have to be changed. It is easier to use a software operating with FEM, which can determine both solutions in one computation step. When the structure is more complicated, the analytic calculation becomes almost unable to achieve.

\subsection{Results of Simulation}

A simulation in COMSOL Multiphysics 5.0 (COMSOL Inc.) was performed and the results were compared with the analytic equation. The simulation utilized MUMPS Eigenfrequency solver and was focused on frequencies in the range from 0 to $6 \mathrm{kHz}$, which can also be measured with an accessible equipment and compared. Dimensions of the tested beam are $40 \times 4 \times 1 \mathrm{~cm}$ and the largest mesh element size was chosen as $0.4 \mathrm{~cm}$, therefore, on the edges of the object, there are $101 \times 11 \times 4$ nodes. For a verification, a comparison was made with a half mesh density, the error of the determined frequency in the examined frequency range was less than $0.55 \%$. A further comparison was made with a double mesh density and the error of the determined frequencies was less than $0.06 \%$. The considered computing mesh is a suitable compromise between the computing time and the quality of the result for future simulations.

Shapes of some modes are shown in Figure 1. Computed resonance frequencies are shown in Table 2 Parameters: $L=0.4 \mathrm{~m}, b=0.04 \mathrm{~m}, h=0.01 \mathrm{~m}$, $E=2 \cdot 1011 \mathrm{~Pa}, \rho=7870 \mathrm{~kg} / \mathrm{m}^{3}$.

\section{MATERIALS AND METHODS}

As was mentioned in Introduction, the aim of this study is to perform two methods of modal analysis and compare them. One method uses one sensor and structure is excitated in many different points, the other method applies laser interferometry and structure is multi-point measured.

\subsection{STATIC SENSOR}

In the first experiment, a modal hammer Endevco 2302-5 was used for exciting the structure in a created mesh of 27 points $(3 \times 9)$. It is able to excite frequencies up to $8 \mathrm{kHz}$ with the aluminium tip and its $100 \mathrm{~g}$ head mass. The chosen mesh is sufficient to measure up to 7 longitudinal modes and based on the simulation, this should cover a frequency range up to $8 \mathrm{kHz}$. However, this is the uttermost condition, the resulting animation will not look smooth and it was chosen as a compromise between the amount of captured data and the quality of the result.

A small accelerometer Metra KD91 was used as a sensor, complemented with a charge amplifier and the signal was processed by 24-bit acquisition module NI9234. The sensor was placed in a corner of a structure to guarantee the measurement of all eigenfrequencies influencing Z-axis vibrations. The weight of the sensor is $1.8 \mathrm{~g}$, it is negligible in comparison with the mass of the beam $(\sim 1.3 \mathrm{~kg})$. The measured beam was placed on a soft foam pad which represents a free boundary conditions.

\subsection{Static excitation}

For the second experiment, laser interferometer Polytec OFV-5000 with a custom 2D extension was used, the response can be measured in more points with 


\begin{tabular}{ccc}
\hline & \multicolumn{2}{c}{ frequency $(\mathrm{Hz})$} \\
\cline { 2 - 3 } longitudinal mode n. & analytic equation & FEM \\
\hline 1 & 323.9 & 323.3 \\
2 & 892.8 & 888.3 \\
3 & 1750 & 1733 \\
4 & 2893 & 2848 \\
5 & 4322 & 4220 \\
\hline diagonal mode n. & analytic equation & FEM \\
\hline 1 & & 1751 \\
2 & not calculated & 3520 \\
3 & & 5324 \\
\hline
\end{tabular}

TABLE 2. Computed resonance frequencies.

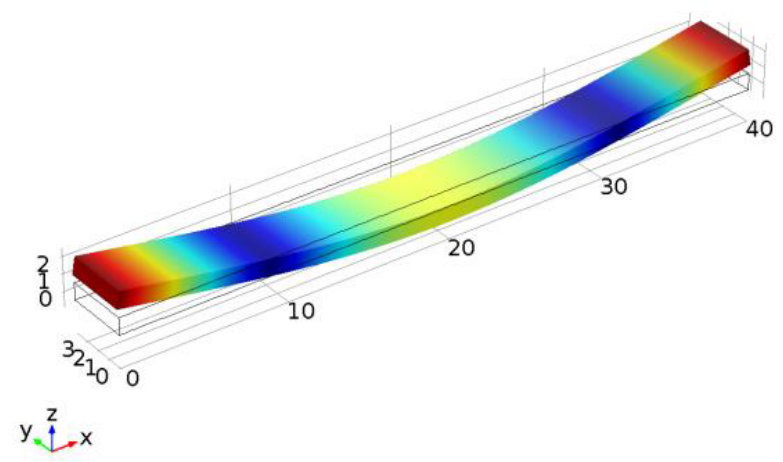

(A).

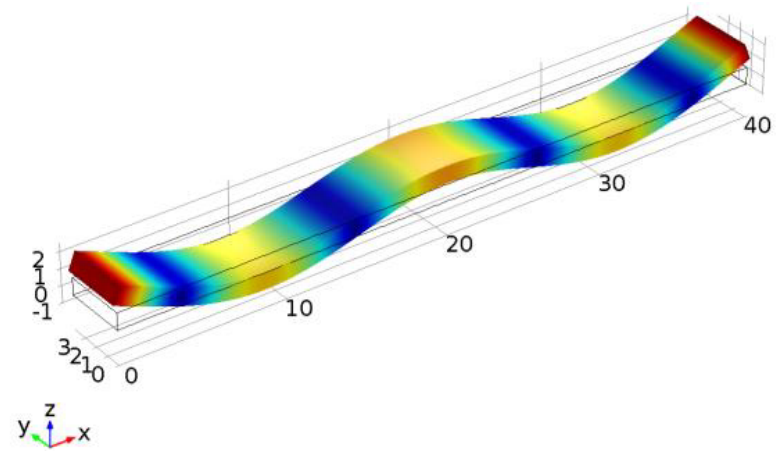

(c).

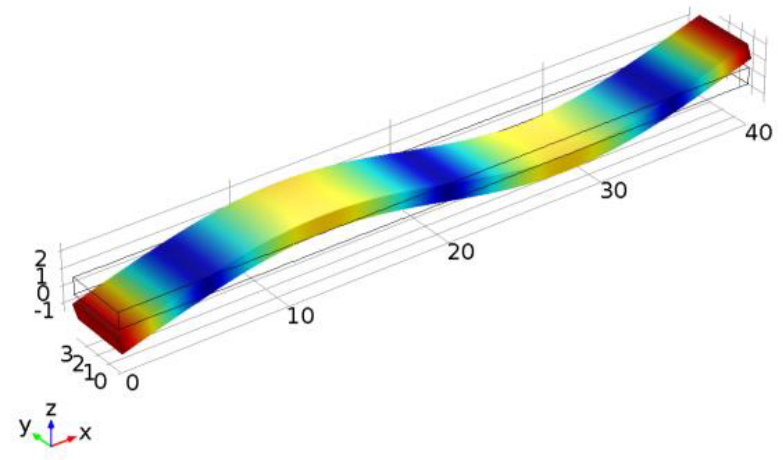

(B).

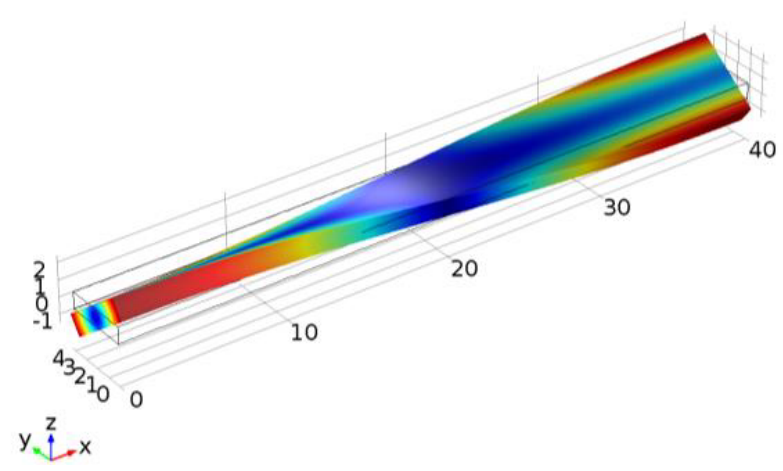

(D).

Figure 1. Shapes of some selected modes ((A)-(C) - three longitudinal modes, (D) - diagonal mode). 


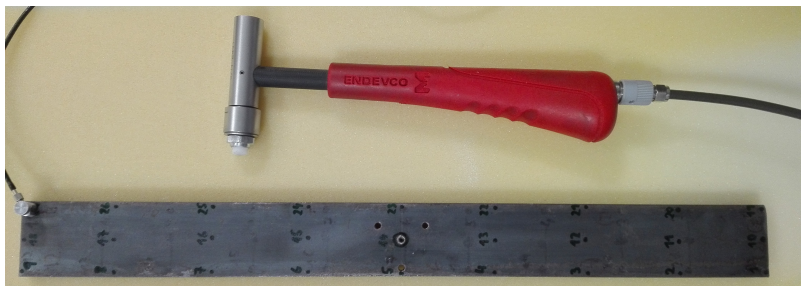

FiguRE 2. Modal hammer and examined structure with attached sensor.

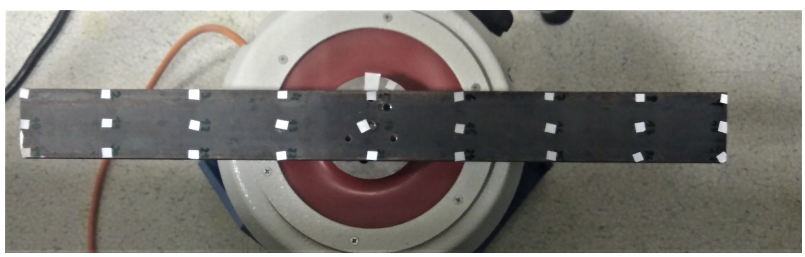

FIGURE 3. Examined structure attached to vibration test system.

higher certainty of the measured point and the structure is not influenced by the sensing element. The same 27-point grid of measuring points was chosen for the measurement, to provide the most matching comparison with the previous method.

A vibration test system Tira S 52110 was used for the excitation of the beam. The generator was operating with a random noise signal and chirp sinusoidal signal. Both of the signals need to be limited due restrictions of the vibration system (limitations in frequency range and amplitude). Eventually a chirp signal was chosen as more suitable for its better results, the random noise signal did not have a sufficient amplitude to visualize all modes with better precision than the chirp signal. In addition, the noise signal is less user friendly, which is also a key factor for the creation of a laboratory set for students.

\subsection{SignAL PROCESSING}

This arrangement was processed by ABSignal ModalVIEW (National Instruments), which is developed for performing modal analyses. It allows defining the measured structure, setting measurement and equipment and computes FRF (frequency response function).

Both methods were processed with the same signal processing chain. For the FFT analysis, the sampling frequency was chosen as $51.2 \mathrm{kHz}$ and a rectangle window was applied, the measurement in each point of the structure composes of three measurements and averaging the RMS. Mode estimation utilizes the LSCF algorithm.

\section{Results}

Main resonant frequencies are obvious from the measured FRF in Figure 4 but in the area around $1800 \mathrm{~Hz}$, two peaks are noticeable. It may be hard to determine, without further knowledge, if it is an error and it belongs to one mode, or they signify two different modes. In the context of simulation, it is easier to determine the relationship to two different modes. This feature shows one disadvantage of the measurement. Two different modes can be located at nearby frequencies and a less experienced operator may misinterpret the results. Therefore, it is advisable to perform a simulation before the measurement itself.

Experimental results have been compared against the analytical results in Table 3 The difference between the measurement and computed frequencies was 1 to $4 \%$, between the measurement and frequencies by the FEM from 3 to $4 \%$, so these values are not so accurate when compared to the equation, but more consistent, which can ïndicate a lack of knowledge about material properties.

The results of the experiment with static excitation showed some similar outcomes, but many of them vary more than those with the static sensor (Figure 4). The fixed connection between the measuring system and the beam is the main reason, which shifts many of the Eigenfrequencies to lower values.

A simulation for an asymmetric attachment showed different results of modal shapes in comparison with free boundary conditions. Many of them have a different amplitude of antinodes on the opposite side of the beam and for some of them, it is hard to recognize the longitudinal or diagonal mode. An example of a shape with the asymmetric attachment is shown in Figure 5 With the symmetric attachment, the shapes are similar to the free arrangement.

The attachment caused a shift of the frequencies of odd modes to lower values and of the even modes to higher compared with results in the free arrangement. Simulation also showed some resonances, which were not present in the basic variety. Results are summed up in Table 4

Apart from the first longitudinal mode, where the error reached $40 \%$, which was most probably caused by the influence of the vibration system and inertia of the beam, the error between the measured frequencies and those determined by the FEM simulation were in a range from 1 to $6 \%$. The comparison with the analytic solution is not suitable in this case, because the boundary conditions are changed and they are not covered in the equation.

To summarize the results, even though the parameters in the calculations were same, the results are slightly different, but the maximum error is approximately $2.5 \%$. The data achieved by the experiment vary more from the results of the simulation, but this may be caused by a lack of knowledge about the material properties.

\section{DisCUSSION}

One of the main focus of this article was a comparison of two procedures of modal analysis and their comparison. First applied method - roving excitation and static sensing - can be determined as a relatively cheap and quickly prepared. One of disadvantages of 

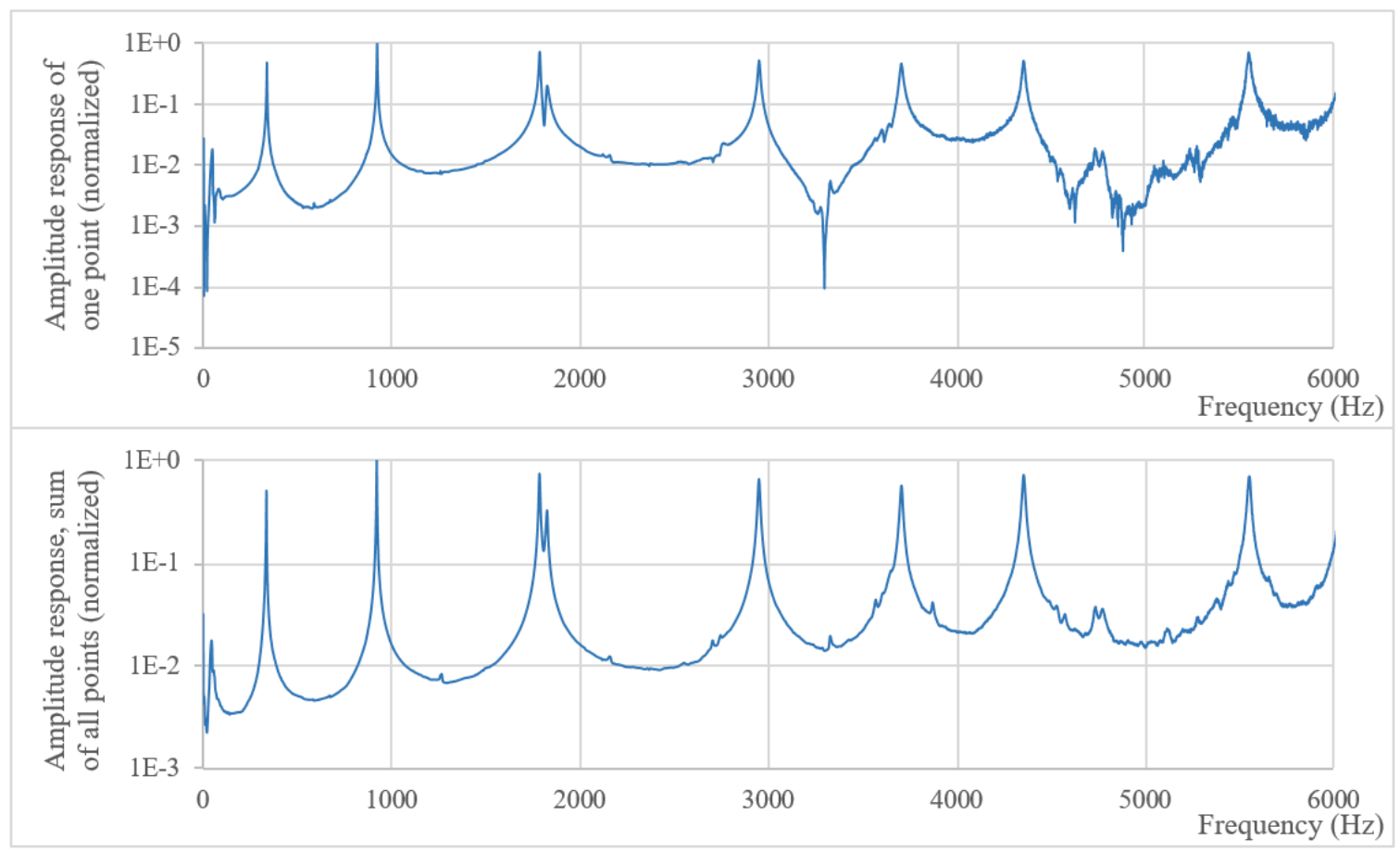

FiguRE 4. Measured FRF in a range up to $6 \mathrm{kHz}$ by static sensor. Up - FRF from 1 measurement. Down - Sum of all measurements.

\begin{tabular}{cccc}
\hline & \multicolumn{3}{c}{ frequency $(\mathrm{Hz})$} \\
\cline { 2 - 4 } & \multicolumn{2}{c}{ compute } & measurement \\
\hline longitudinal mode n. & equation & FEM & static sensor \\
\hline 1 & 324 & 323.3 & 336 \\
2 & 893 & 888.4 & 921 \\
3 & 1750 & 1734 & 1784 \\
4 & 2893 & 2849 & 2949 \\
5 & 4322 & 4222 & 4354 \\
\hline diagonal mode n. & equation & FEM & static sensor \\
\hline 1 & & 1775 & 1826 \\
2 & not calculated & 3567 & 3705 \\
3 & & 5395 & 5550 \\
\hline
\end{tabular}

TABLE 3. Comparison of model and experiment with static sensor.

\begin{tabular}{cccc|cr}
\hline & \multicolumn{3}{c}{ frequency $(\mathrm{Hz})$} \\
\cline { 2 - 5 } & \multicolumn{3}{c}{ FEM compute } & \multicolumn{2}{c}{ measurement } \\
\cline { 2 - 5 } & free arrangement & sym. attach. & asym. attach. & sym. attach. & asym. attach. \\
\hline \multirow{5}{*}{ longitudinal modes } & 323.3 & 204.2 & 207.7 & 292.4 & 293.6 \\
& 888.3 & 1018 & 980.4 & 953.2 & 931.5 \\
& 1734 & 1270 & 1296 & 1300 & 1554 \\
& 2849 & 3004 & 2864 & 2952 & 2833 \\
diagonal modes & 4222 & 3490 & 3361 & 3662 & 3241 \\
& - & 1320 & 1386 & 1350 & 1649 \\
& 1775 & 1753 & 1771 & 1699 & 1825 \\
& 3567 & 4242 & 4172 & 4191 & 3994 \\
\hline
\end{tabular}

TABLE 4. Comparison of model and experiment with static excitation. 


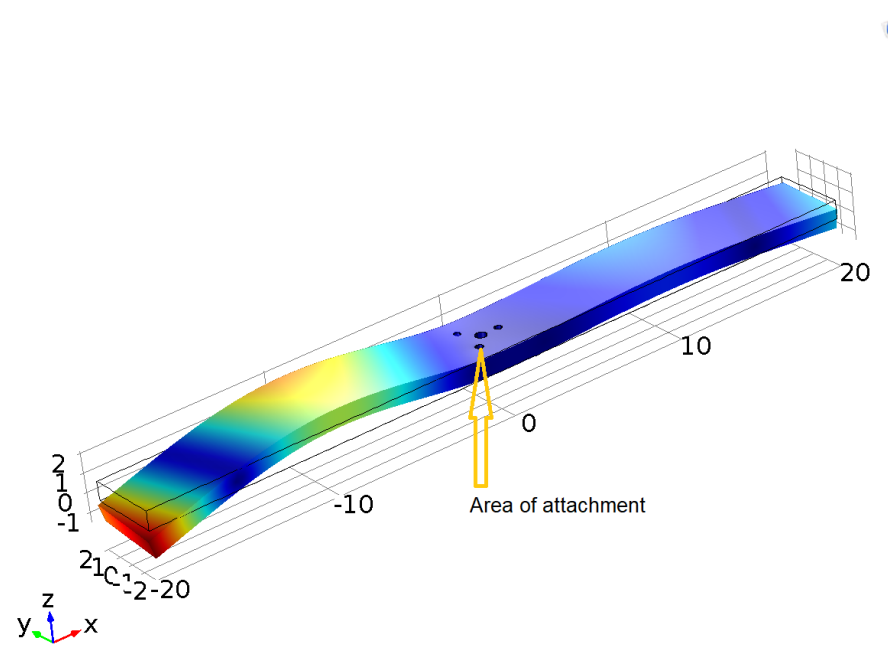

FIGURE 5. Modal shape with asymmetric attachment.
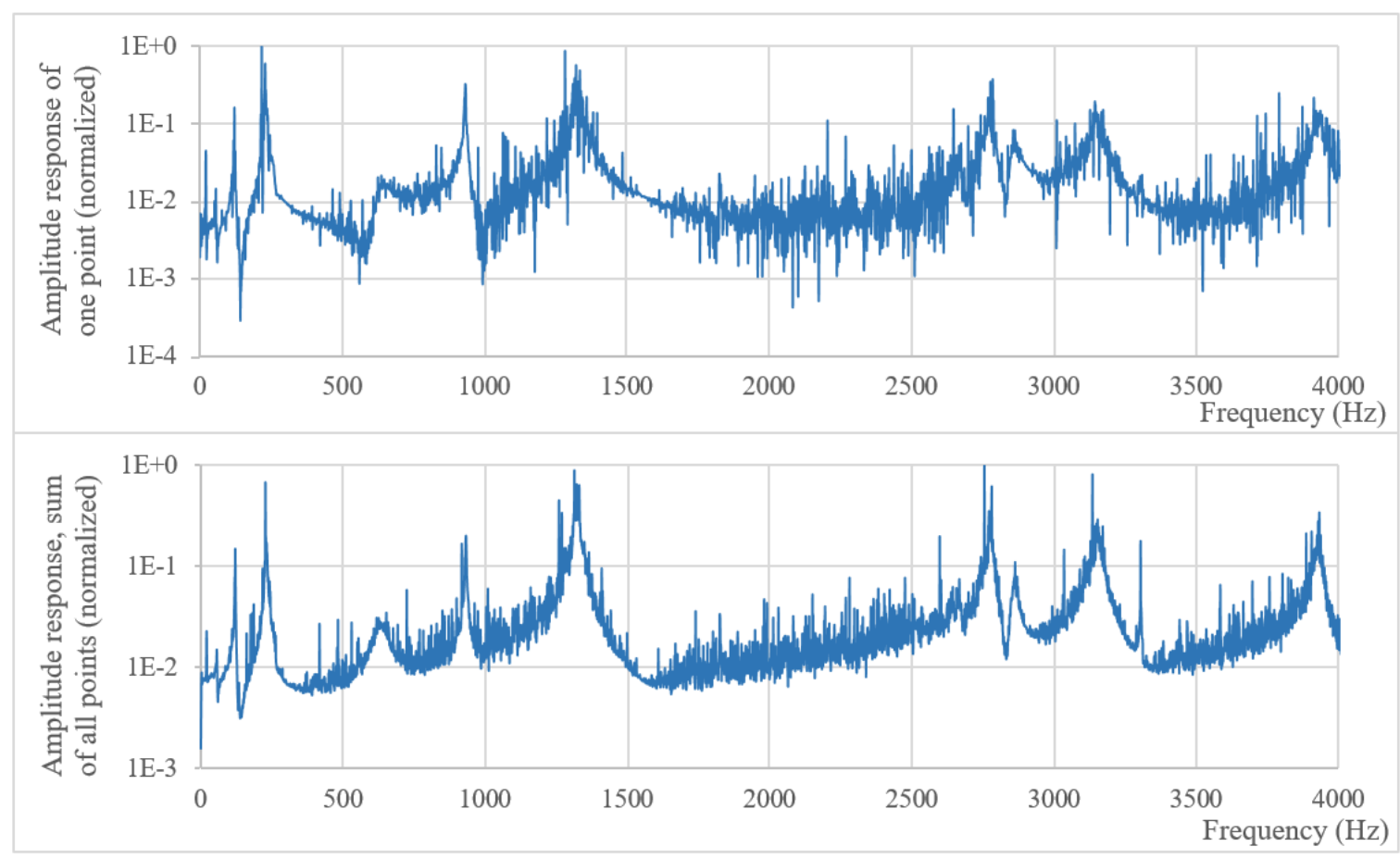

FigURE 6. Measured FRF in a range up to $4 \mathrm{kHz}$ by static excitation with chirp sinusoidal signal. Up - FRF from 1 measurement. Down - Sum of all measurements. 
this method is the dependency on operator, because he is controlling the measurement and the excitation manually. Moreover, the accelerometer adds mass to the beam and it can influence the measurement. In this case, the accelerometer is $1000 \times$ lighter than the beam, it does not affect the results. In comparison with the analytic solution and simulation, some differences originating from material constants and design of free arrangement can be found - the usage of the soft foam pad does not fully represent a free attachment.

Unfortunately, the method with the static excitation has more disadvantages then the previous one. The biggest disadvantage of this arrangement, which significantly showed during the measurement, is the influence of the vibration system. It can cause additional vibrations or change the resonance frequencies. The measured structure was small in comparison with the vibrating system and the beam was tightly connected to the vibration system by a bolt. Therefore, this arrangement cannot be considered as free and the FEM model had to be redone to match this situation. The method with the static excitation is commonly used, for example in automotive industry for bodywork analysis, but the vibration system is smaller in comparison with the measured structure and connected by a rod.

Another disadvantage is the choice of a place for the excitation, which is similar to the sensing point in the previous method. Firstly, the attachment to the centre did not seem as suitable, because some modes do not have to vibrate due the attachment in the nodes. Therefore, two different attachments were used to determine this influence. Finally, the attachment to the centre was applicable, all modes were able to be measured and the asymmetric attachment caused changes of the measured shape to irregular, it significantly modified the examined situation.

This set is much more expensive and demanding on preparation, because reflexive elements must be placed in each point of measurement. This method is less prone to operator failures and the measurement is autonomous after the set up. It could also be carried out with a modal hammer and accelerometer, but changing the position of the accelerometer is very demanding, predisposed to some mistakes and it modifies the structure between measurements. Thus it can be described as doable, but unsuitable.

The repeatability of the measurement with the static sensor and modal hammer is lower than $0.5 \%$ with different interdependent operators and error between calculations and experiments was lower than $4 \%$. The repeatability of the measurement with the static excitation and interferometer was less than $0.2 \%$, but more inaccurate $(40 \%$ error for first mode and under $6 \%$ for higher modes) and not so consistent. The main reason is the influence of the vibration system and fixed connection between the structure and the excitation system. Therefore, the preparation and utilization of a small periodical autonomous modal hammer for the static excitation is planned for further experiments using the static excitation and roving sensing.

\section{Conclusion}

This paper presented a modal analysis of a basic beam in a free arrangement, which was performed and compared to resonance frequencies determined numerically. Two different procedures of mode estimation were compared from different points of view.

Based on the mentioned arguments and better results, the method with a static sensor and rowing excitation with a modal hammer was chosen for purposes of a laboratory set for students. This method uses cheaper equipment, and thus it is more accessible. This type of measurement is more suitable for structures of smaller dimensions like the examined one, even though the repeatability of the measurement is lover and the measurement is not autonomous. The design of the measuring procedure with the static excitation is more crucial than that of the method with the roving excitation.

\section{ACKNOWLEDGEMENTS}

The completion of this paper was made possible by the grant No. FEKT-S-17-4234 - „Industry 4.0 in automation and cybernetics" financially supported by the Internal science fund of Brno University of Technology.

\section{LIST OF SYMBOLS}

$L$ length of beam $[\mathrm{m}]$

$\rho$ density $\left[\mathrm{kg} \mathrm{m}^{-3}\right]$

$E$ Young's modulus [Pa]

$I$ cross section moment of area $\left[\mathrm{m}^{4}\right]$

$A$ area of cross section $\left[\mathrm{m}^{2}\right]$

$w$ transfer displacement $[\mathrm{m}]$

$t$ time [s]

$b$ beam width $[\mathrm{m}]$

$h$ beam height $[\mathrm{m}]$

\section{REFERENCES}

[1] S. E. Haji Agha Mohammad Zarbaf, R. Allemang. A modification to unified matrix polynomial approach (UMPA) for modal parameter identification. In C. Niezrecki, J. Baqersad (eds.), Structural Health Monitoring, Photogrammetry \& DIC, Volume 6, pp. 1-8. Springer International Publishing, Cham, 2019. DOI:10.1007/978-3-319-74476-6_1.

[2] R. J. Allemang, D. L. Brown. A unified matrix polynomial approach to modal identification. Journal of Sound and Vibration 211(3):301 - 322, 1998. DOI:10.1006/jsvi.1997.1321

[3] J. M. M. Silva, N. M. M. Maia. Modal Analysis and Testing. Springer, 1999, Netherlands.

[4] J. He, Z.-F. Fu. Modal Analysis.

Butterworth-Heinemann, 2001. 
[5] D.-T. He, Y. Guo. Finite element analysis of humanoid robot arm. In 13th International Conference on Ubiquitous Robots and Ambient Intelligence, pp. 772 776. 2016. DOI:10.1109/URAI.2016.7733979

[6] M. Imran, A. A. Abbasi, M. J. Hyder. Determination of modal characteristics of cantilever beam. In International Conference on Emerging Technologies, pp. 1 - 3. 2016. DOI:10.1109/ICET.2016.7813224.

[7] B. Zheng, J. Zhang, Y. Yao. Finite element analysis of the piston based on ANSYS. In IEEE 3rd Information Technology, Networking, Electronic and Automation Control Conference, pp. 1908 - 1911. 2019. DOI:10.1109/ITNEC.2019.8729409.

[8] S. Qi. Modal test analysis of prestressed concrete simply supported beam bridge. In International Conference on Robots $\&$ Intelligent System, pp. 86 - 89 . 2018. DOI:10.1109/ICRIS.2018.00030.
[9] E. Orlowitz, A. Brandt. Comparison of experimental and operational modal analysis on a laboratory test plate. Measurement 102:121 - 130, 2017. DOI:10.1016/j.measurement.2017.02.001

[10] V. Ondra, B. Titurus. Theoretical and experimental modal analysis of a beam-tendon system. Mechanical Systems and Signal Processing 132:55 - 71, 2019. DOI:10.1016/j.ymssp.2019.06.016

[11] M. Avcar. Free vibration analysis of beams considering different geometric characteristics and boundary conditions. International Journal of Mechanics and Applications 4(3):94 - 100, 2014. DOI:10.5923/j.mechanics.20140403.03

[12] L. Meirovitch. Fundamentals of Vibrations. McGraw-Hill, 2001.

[13] L. H. Donnell. Beams Plates and Shells, Engineering Societies Monographs. McGraw-Hill, 1976. 